PROCEEDINGS OF THE

AMERICAN MATHEMATICAL SOCIETY

Volume 131, Number 3, Pages 979-989

S 0002-9939(02)06605-4

Article electronically published on October 18, 2002

\title{
ON ULTRAMETRIZATION OF GENERAL METRIC SPACES
}

\author{
ALEX J. LEMIN
}

(Communicated by Alan Dow)

\begin{abstract}
This paper gives a complete description of ultrametric spaces up to uniform equivalence. It also describes all metric spaces which can be mapped onto ultrametric spaces by a non-expanding one-to-one map. Moreover, it describes particular classes of spaces, for which such a map has a continuous (uniformly continuous) inverse map. This gives a complete solution for the Hausdorff-Bayod Problem (what metric spaces admit a subdominant ultrametric?) as well as for two other problems posed by Bayod and Martínez-Maurica in 1990. Further, we prove that for any metric space $(X, d)$, there exists the greatest non-expanding ultrametric image of $X$ (an ultrametrization of $X$ ), i.e., the category of ultrametric spaces and non-expanding maps is a reflective subcategory in the category of all metric spaces and the same maps. In Section II, for any cardinal $\tau$, we define a complete ultrametric space $L_{\tau}$ of weight $\tau$ such that any metric space $X$ of weight $\tau$ is an image of a subset $L(X)$ of $L_{\tau}$ under a non-expanding, open, and compact map with totally-bounded pre-images of compact subsets. This strengthens Hausdorff-Morita, Morita-de Groot and Nagami theorems. We also construct an ultrametric space $L(\tau)$, which is a universal pre-image of all metric spaces of weight $\tau$ under nonexpanding open maps. We define a functor $\lambda$ from the category of ultrametric spaces to a category of Boolean algebras such that algebras $\lambda(X)$ and $\lambda(Y)$ are isomorphic iff the completions of spaces $X$ and $Y$ are uniformly homeomorphic. Some properties of the functor $\lambda$ and the ultrametrization functor are discussed.
\end{abstract}

A metric space is called ultrametric (non-Archimedean in German literature, isosceles in Russian) if its metric satisfies the strong triangle axiom $d(x, z) \leq$ $\max [d(x, y), d(y, z)]$.

The first significant examples of such spaces were introduced in early 20th century in real analysis (René Baire, 1909, the Baire space), number theory (Kurt Hensel, 1904, rings $\mathbf{Q}_{p}$ and fields $\mathbf{Z}_{p}$ of $p$-adic numbers), and general topology (Felix Hausdorff, 1914, 1930). In [7], [8] F. Hausdorff studied their properties (in separable case) and their relation to general metric spaces. What metric spaces are images and pre-images of non-Archimedean spaces under particular classes of continuous maps (open, closed, compact, uniformly continuous, non-expanding, etc.)? This paper gives a complete solution of the Hausdorff problem for classes of non-expanding

Received by the editors December 30, 2000 and, in revised form, October 29, 2001.

2000 Mathematics Subject Classification. Primary 54E35, 54E05, 54E40, 54E50; Secondary 06B30, 06E15, 11E95, 12J25, 18A40, 18B30, 26E30, 54B30, 54C10, 54D30.

Key words and phrases. Metric space, ultrametric space, proximity space, complete space, compactification, Smirnov compactification, uniform equivalence, non-expanding map, subdominant ultrametric, ultrametrization of metric space, Boolean algebra, category, functor, reflective functor, ultrametrization functor, isomorphism of categories.

(C)2002 American Mathematical Society 
maps and uniformly continuous maps (by the definition, a map $f:(X, d) \rightarrow\left(Y, d^{\prime}\right)$ is non-expanding if it enlarges no distance, i.e., $d(x, y) \geq d^{\prime}(f(x), f(y))$ for any $x$ and $y$ in $X$ ). Section $\square$ deals with pre-images of ultrametric spaces and Section $\amalg$ with images.

\section{NON-EXPANDING PRE-IMAGES OF ULTRAMETRIC SPACES}

Let $(X, d)$ be a metric space. For non-expanding one-to-one maps, the Hausdorff Problem is equivalent to the following question. Does there exist an ultrametric $\Delta(x, y)$ smaller than $d(x, y)$ ? If so, there obviously exists the greatest of such ultrametrics since a supremum of all ultrametrics smaller than $d$ is an ultrametric again; this is called a subdominant ultrametric 11. The problem of existence of the subdominant ultrametric also arises in certain branches of physics [23]. For finite spaces this does exist, moreover, some computational algorithms are known [23].

In [1] J. M. Bayod and J. Martínez-Maurica studied the problem for infinite spaces and gave a sufficient condition for existence of a subdominant ultrametric. In view of what was mentioned above, the Hausdorff-Bayod Problem can be stated as follows.

Problem 1. For what metric spaces $(X, d)$, does there exist an ultrametric $\Delta(x, y)$ such that the identity map $i:(X, d) \rightarrow(X, \Delta)$ is non-expanding?

Theorem 1 below completely solves this problem, moreover, it describes particular classes of spaces for which an inverse map $i^{-1}:(X, \Delta) \rightarrow(X, d)$ is $(1)$ one-to-one, (2) continuous, (3) uniformly continuous. To introduce these spaces we recall basic notions of the theory of proximity spaces following the original article by Efremovich [4], Yuri Smirnov's fundamental papers [24]-26] and our note [10].

A sequence of points $a=x_{0}, x_{1}, \ldots, x_{n-1}, x_{n}=b$ in a metric space $(X, d)$ is called an $\varepsilon$-chain joining $a$ and $b$ if $d\left(x_{k}, x_{k+1}\right) \leq \varepsilon$ for any $k \leq n$. A space $(X, d)$ is Cantor connected [9, [4] (or pre-connected [1]) if any two points can be joined by an $\varepsilon$-chain for any $\varepsilon>0$. For any point $a$ in $X$ there is a greatest Cantor connected subset $[a]$ containing a (a component of Cantor connectedness of a). Efremovich proved [4] that $(X, d)$ is Cantor connected if and only if it cannot be partitioned into two non-empty remote parts. For metric proximity, two subsets $A$ and $B$ are remote, $A \bar{\delta} B$, if $\operatorname{dist}(A, B)=\inf \{d(x, y) \mid x \in A, y \in B\}>0$; otherwise they are close, $A \delta B$. The classes of proximity spaces we are in need of are introduced by an analogy with the well-known classes of topological spaces.

\begin{tabular}{|c|c|}
\hline $\begin{array}{l}\text { (0) } X \text { is totally disconnected, } \\
\text { i.e., there is no connected, } \\
\text { non-singleton subsets in } X\end{array}$ & $\begin{array}{l}\left(0^{\prime}\right) X \text { is totally Cantor disconnected, } \\
\text { i.e., there is no Cantor connected } \\
\text { non-singleton subsets in } X\end{array}$ \\
\hline $\begin{array}{l}\text { (1) For any two points } a \neq b \text { in } X \\
\text { there is } X_{1} \ni a \text { such that } X_{2}=X \backslash X_{1} \ni b \\
\text { and both } X_{1} \text { and } X_{2} \text { are open and closed }\end{array}$ & $\begin{array}{l}\left.\text { (1 } 1^{\prime}\right) \text { For any two points } a \neq b \text { in } X \\
\text { there is } X_{1} \ni a \text { such that } X_{2}=X \backslash X_{1} \ni b \\
\text { and } X_{1} \text { and } X_{2} \text { are remote, } X_{1} \bar{\delta} X_{2}\end{array}$ \\
\hline $\begin{array}{l}\text { (2) } X \text { is small inductive } \\
\text { zero-dimensional, ind } X=0\end{array}$ & $\begin{array}{l}\left(2^{\prime}\right) X \text { is small proximate zero-dimensional, } \\
\text { in } \delta X=0 \text {, that is, for any } a \text { in } X \\
\text { and } A \subset X \text { such that } a \bar{\delta} A \text { there is } X_{1} \ni a \\
\text { such that } X_{2}=X \backslash X_{1} \supset A \text { and } X_{1} \bar{\delta} X_{2}\end{array}$ \\
\hline $\begin{array}{l}\text { (3) } X \text { is large inductive } \\
\text { zero-dimensional, Ind } X=0 \\
\text { (for metric spaces, this is } \\
\text { equivalent to zero-dimensionality } \\
\operatorname{dim} X=0 \text { in the Lebesgue sense) }\end{array}$ & $\begin{array}{l}\left(3^{\prime}\right) X \text { is large proximate } \\
\text { zero-dimensional, In } \delta X=0 \text {, that is, } \\
\text { for any remote subset } A \text { and } B \text { in } X \text { there } \\
\text { is } X_{1} \supset A \text { such that } X_{2}=X \backslash X_{1} \supset B \\
\text { and } X_{1} \bar{\delta} X_{2}\end{array}$ \\
\hline
\end{tabular}


The following lemmas [10] are easy to prove.

Lemma 1. All properties $\left(0^{\prime}\right)-\left(3^{\prime}\right)$ are hereditary.

Lemma 2. Large proximate zero-dimensionality, In $\delta X=0$, is equivalent to the proximate zero-dimensionality in the sense of Smirnov, $\delta \operatorname{dim} X=0$, [10, [26].

The latter is equivalent [26] to a zero-dimensionality $\operatorname{dim} \sigma X=0$ of the Smirnov compactification $\sigma X$ of a given proximity space $(X, \delta)$ (= a compactification that corresponds to a given proximity $\delta$ [24]).

Lemma 3. Like the well-known implications $(3) \Rightarrow(2) \Rightarrow(1) \Rightarrow(0)$, the following implications are true: $\left(3^{\prime}\right) \Rightarrow\left(2^{\prime}\right) \Rightarrow\left(1^{\prime}\right) \Rightarrow\left(0^{\prime}\right)$; also $\left(1^{\prime}\right) \Rightarrow(1)$ and $\left(2^{\prime}\right) \Rightarrow(2)$.

Note that in general $\left(3^{\prime}\right)$ does not imply (3). The Tykhonoff plane, $T=\left[1, \omega_{0}\right]$. $\left[1, \omega_{1}\right] \backslash\left(\omega_{0}, \omega_{1}\right)$ is proximate zero-dimensional, $\delta \operatorname{dim} T=0$, but not normal, thus $\operatorname{dim} T \neq 0[5]$.

Lemma 4. For compacta, $\left(3^{\prime}\right) \Rightarrow(3)$ and all the properties $(0)-(3),\left(0^{\prime}\right)-\left(3^{\prime}\right)$ are equivalent.

Example 1 below shows that each of the classes $\left(0^{\prime}\right)-\left(3^{\prime}\right)$ strictly contains the next one even in case of metrizable proximity spaces (even among subsets of a Euclidean plane!).

Lemma 5. For metric spaces, the properties $\left(1^{\prime}\right)-\left(3^{\prime}\right)$ are respectively equivalent to the following:

$\left(1^{\prime \prime}\right)$ For any two points $a \neq b$ in $X$ there is $\varepsilon>0$ such that $a$ and $b$ cannot be joined by an $\varepsilon$-chain ( $a$ and $b$ are not $\varepsilon$-linkable). We call these spaces totally unlinked (10 $)$.

$\left(2^{\prime \prime}\right)$ For any point a in $X$ and any subset $A$ such that $a \bar{\delta} A$ there is $\varepsilon>0$ such that $a$ and $A$ cannot be joined by an $\varepsilon$-chain.

$\left(3^{\prime \prime}\right)$ For any two remote subsets $A$ and $B$ in $X$ there is $\varepsilon>0$ such that $A$ and $B$ cannot be joined by an E-chain.

Example 1. Let $X$ be a subset of a Euclidean plane,

$$
X=(0,0) \cup(0,1) \cup\{(1 / n, m / n) \mid n, m \in \mathbf{N}, 0 \leq m \leq n\} .
$$

$X$ is totally Cantor disconnected but the points $(0,0)$ and $(0,1)$ are $\varepsilon$-linkable for any $\varepsilon>0((0,0)$ and $(0,1)$ are linked $)$. Thus $X$ does not belong to $\left(1^{\prime}\right)$.

Let $X^{\prime}=X \backslash(0,1)$. $X^{\prime}$ is totally unlinked, a point $(0,0)$ and a subset $A=$ $\left\{(x, y) \in X^{\prime} \mid y=1\right\}$ are remote. However they are linked. Thus $X^{\prime}$ does not belong to $\left(2^{\prime}\right)$.

Let $X^{\prime \prime}=X^{\prime} \backslash(0,0)$. The subsets $A$ and $B=\left\{(x, y) \in X^{\prime \prime} \mid y=0\right\}$ are remote but they are linked. Thus $X^{\prime \prime}$ belongs to $\left(2^{\prime}\right)$ but does not belong to $\left(3^{\prime}\right)$.

Theorem 1. Let $(X, d)$ be a metric space.

(1) If there exists an ultrametric $\Delta(x, y)$ such that the identity map $i:(X, d) \rightarrow$ $(X, \Delta)$ is non-expanding then $(X, d)$ is totally unlinked, and vise versa.

(2) If, in addition, the inverse map $i^{-1}:(X, \Delta) \rightarrow(X, d)$ is continuous, then $X$ is small proximate zero-dimensional, and vise versa.

(3) If, in addition, the inverse map $i^{-1}:(X, \Delta) \rightarrow(X, d)$ is uniformly continuous, then $X$ is large proximate zero-dimensional, and vise versa. 
Proof. First of all we prove that ultrametric spaces satisfy properties $\left(1^{\prime}\right)-\left(3^{\prime}\right)$. In these spaces, the property even stronger than $\left(1^{\prime}\right)$ holds: no two different points a and $b$ in $X$ are $\varepsilon$-linkable for any $\varepsilon<d(a, b)$. This property completely characterizes ultrametric spaces.

Lemma 6. A metric space $(X, d)$ is ultrametric if and only if no two different points $a$ and $b$ in it are $\varepsilon$-linkable for any $\varepsilon<d(a, b)$.

The proof is by induction over a length of chain.

From this it easily follows that ultrametric spaces satisfy $\left(2^{\prime}\right)$ and $\left(3^{\prime}\right)$. The sufficiency of the conditions of the theorem now follows from the next lemma and the obvious fact that non-expanding maps are uniform.

Lemma 7. The properties of being totally unlinked (small or large proximate zerodimensional, respectively) are inherited by the inverse images under uniform bijections (uniform homeomorphisms, mutually uniform homeomorphisms, respectively).

Proof. Let $f:(X, d) \rightarrow\left(X, d^{\prime}\right)$ be a uniform bijection, $a \neq b \in X$, and let $\left(X, d^{\prime}\right)$ be totally unlinked. Then there is $X_{1} \ni f(a)$ such that $X_{2}=X \backslash X_{1} \ni f(b)$, and $X_{1}$ and $X_{2}$ are remote in $\left(X, d^{\prime}\right)$. In view of the basic Efremovich Theorem [4], for metric spaces, proximity theory is equivalent to uniformity theory, in particular, a map $f$ is proximate continuous if and only if it is uniformly continuous. Thus $f$ takes close subsets to close ones, so $f^{-1}\left(X_{1}\right) \ni a$ and $f^{-1}\left(X_{2}\right) \ni b$ are remote in $(X, d)$. Hence $(X, d)$ is totally unlinked.

Suppose $f^{-1}:\left(X, d^{\prime}\right) \rightarrow(X, d)$ is continuous, in $\delta\left(X, d^{\prime}\right)=0$, and $a \in X$ and $A \subset$ $X$ are remote in $(X, d)$. This implies that $a \notin[A]_{(X, d)}$. Hence $f(a) \notin[f(A)]_{\left(X, d^{\prime}\right)}$, i.e., $f(a)$ and $f(A)$ are remote in $\left(X, d^{\prime}\right)$. Consequently, there is $X_{1} \ni f(a)$ such that $X_{2}=X \backslash X_{1} \supset f(A)$, and $X_{1}$ and $X_{2}$ are remote in $\left(X, d^{\prime}\right)$. Since $f$ is uniform, $f^{-1}\left(X_{1}\right) \ni a$ and $f^{-1}\left(X_{2}\right) \supset A$ are remote in $(X, d)$. Thus in $\delta(X, d)=0$.

If $f:(X, d) \leftrightarrow\left(X, d^{\prime}\right)$ is uniform in both directions and $\left(X, d^{\prime}\right)$ is proximate zerodimensional, then so is $(X, d)$. Thus Lemma 7 and the sufficiency of the conditions of the theorem are proved.

To prove the necessity we introduce in ANY metric spaces $(X, d)$ a new function $\Delta(x, y)$ smaller than $d(x, y)$ which is a pseudo-ultrametric (i.e., satisfies all axioms of ultrametrics spaces except of the identity axiom " $\Delta(x, y)=0$ implies $x=y$ "). In addition, in cases $\left(1^{\prime}\right),\left(2^{\prime}\right)$ and $\left(3^{\prime}\right)$ it turns out to be an ultrametric such that the inverse map $i^{-1}:(X, \Delta) \rightarrow(X, d)$ is continuous (in case $\left.\left(2^{\prime}\right)\right)$ and uniform (in case $\left(3^{\prime}\right)$ ). Moreover, it is the greatest of such ultrametrics (the latter follows from Theorem 5 below).

Lemma 8. For any metric space $(X, d)$ a function $\Delta(x, y)=\inf \{\varepsilon \mid x$ and $y$ are not $\varepsilon$-linkable\} is a pseudo-ultrametric.

Proof. The properties $\Delta(x, y)=\Delta(y, x), \Delta(x, y) \geq 0$, and $\Delta(x, x)=0$ are obvious. If $x$ and $y$ are $\varepsilon_{1}$-linkable and $y$ and $z$ are $\varepsilon_{2}$-linkable, then $x$ and $z$ are $\max \left[\varepsilon_{1}, \varepsilon_{2}\right]$ linkable. This implies that $\Delta(x, y)$ satisfies the ultrametric axiom.

To complete the proof of Theorem 1 suppose a metric space $(X, d)$ is totally unlinked. Then $\Delta(x, y)>0$ for any $x \neq y$, hence $\Delta$ is an ultrametric.

Suppose in $\delta(X, d)=0$ and $a \delta A$ in $(X, \Delta)$, i.e., $a \in[A]_{(X, \Delta)}$. This means that $\Delta(a, A)=0$, i.e., $a$ and $A$ are linked. This implies that $a \delta A$ in $(X, d)$. Actually, if 
we suppose that $a$ and $A$ are remote in $(X, d)$, then there would be $X_{1} \ni a$ such that $X_{2}=X \backslash X_{1} \supset A$ and $X_{1}$ and $X_{2}$ are remote in $(X, d)$, i.e., $\operatorname{dist}\left(X_{1}, X_{2}\right)>0$. This contradiction shows that $a \delta A$ in $(X, d)$. Therefore, $i^{-1}:(X, \Delta) \rightarrow(X, d)$ is continuous.

Finally, if $\operatorname{In} \delta(X, d)=0$ and $A$ and $B$ are close in $(X, \Delta)$, then $\Delta(A, B)=0$, i.e., $A$ and $B$ are linked. A similar discourse shows that $A$ and $B$ are close in $(X, d)$. Therefore, $i^{-1}:(X, \Delta) \rightarrow(X, d)$ is proximate continuous. In view of the Efremovich Theorem [4, it is uniform.

The first part of the theorem completely solves the Hausdorff-Bayod Problem.

Corollary 1. A metric space $(X, d)$ admits a subdomain ultrametric if and only if it is totally unlinked.

The second part of the theorem describes the spaces which have topologically equivalent subdominant ultrametric and, therefore, answers another problem stated in $[1$.

Problem 2 ([1, p. 831]). Does the existence of subdominant ultrametric guarantee the existence of topologically equivalent ultrametric?

To show that the answer is negative we recall the Morita-de Groot Theorem.

Theorem $([20,6])$. A metric space $(X, d)$ is homeomorphic to an ultrametric one if and only if its zero-dimensional, $\operatorname{dim} X=\operatorname{Ind} X=0$.

In other words, given a metric space $(X, d)$, there is an ultrametric $\Delta(x, y)$ such that the identity map $i:(X, d) \leftrightarrow(X, \Delta)$ is homeomorphic if and only if Ind $X=0$. Thus any totally unlinked metric space of positive dimension (if one exists) would answer Problem 2 in the negative. An example of such space (a onedimensional totally unlinked subset of Hilbert space) was first constructed in 1984 in [10]. Later on J. M. Bayod solved the problem independently by constructing a different example in [2]. These examples also prove the last part of Corollary 2 below.

Theorem 1 can be reformulated as follows, 10 .

Theorem 1'. (1) Totally unlinked metric spaces (and only they) are inverse images of ultrametric spaces under uniform bijections.

(2) Small proximate zero-dimensional metric spaces (and only they) are inverse images of ultrametric spaces under uniform (in one direction) homeomorphisms.

(3) Large proximate zero-dimensional metric spaces (and only they) are inverse images of ultrametric spaces under uniform (in both direction) homeomorphisms.

The third part of the theorem gives a complete description of ultrametric spaces up to uniform equivalence (= proximate equivalence) and implies a few algebraic corollaries.

Corollary 2. For metric spaces as well as for compacta, property $\left(3^{\prime}\right)$ implies property (3) (even (2') implies (3)), however from (1') neither (3) nor (2) follow.

Thus any proximate zero-dimensional metric space is zero-dimensional (in $\delta X=$ 0 implies Ind $X=0$ ). On the other hand, any zero-dimensional metric space is homeomorphic) (but not uniformly homeomorphic, in general) to an ultrametric 
space. Thus ultrametrics are in a certain sense the most natural metrics for zerodimensional spaces: they are those metrics with respect to which a compactification of these spaces is zero-dimensional. A proximity space is called proximate metrizable 25] if there exists a metric that induces a given proximity. Combining Lemma 2 and part (3) of the theorem we get the following.

Corollary 3. From the point of view of proximity geometry, ultrametric spaces are none other than proximate metrizable subsets of zero-dimensional compacta.

Corollary 3 and [14, Theorem $1^{\prime}$ and Corollary 5] combined reduce a uniform study of ultrametric spaces to algebra.

Theorem 2. There exists a contra-variant functor $\lambda$ : Umet $\rightarrow$ Bool from the category of ultrametric spaces and uniformly continuous maps Umet to the category of Boolean algebras Bool such that algebras $\lambda(X)$ and $\lambda(Y)$ are isomorphic if and only if completions of the spaces $X$ and $Y$ are uniformly homeomorphic.

Proof. Consider the following scheme, 17]:

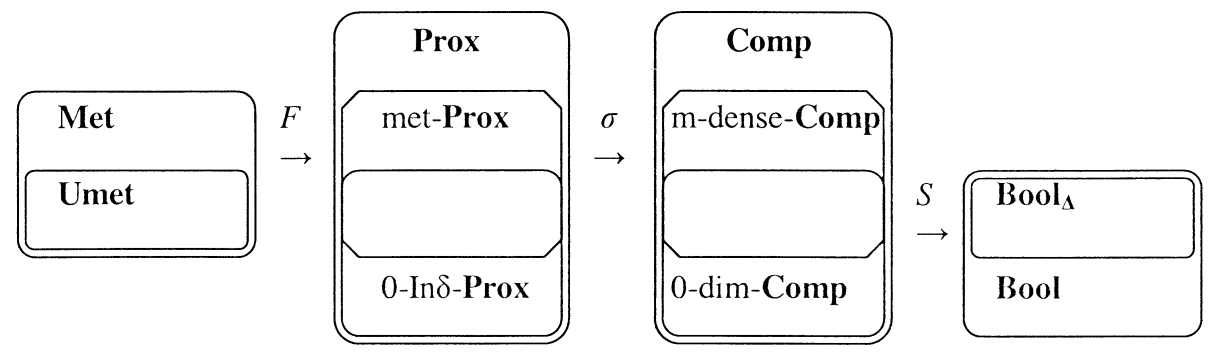

Here Met (Umet) is a category of all metric (ultrametric) spaces and uniform maps. $F$ is a forgetful functor from Met to a category Prox of proximity spaces, and $m$-Prox, 0 - In $\delta$-Prox are categories of proximate metrizable (proximate zerodimensional, respectively) spaces. An intersection of the latter two is the image $F$ (Umet), in view of Theorem 1' (part (3)). Further, Comp (0- dim-Comp, mdense-Comp) denotes a category of compact spaces (zero-dimensional compact spaces, compact spaces with dense metrizable subsets, respectively). $\sigma$ is the Smirnov compactification functor. By Corollary 3, a composition $\sigma \circ F$ maps Umet onto an intersection of 0- dim-Comp and m-dense-Comp. Finally, we use the Marshall Stone duality functor $S$ : 0-dim-Comp $\leftrightarrow$ Bool between zero-dimensional compacta and Boolean algebras [27, [5] and set $\lambda=S \circ \sigma \circ F$.

The Smirnov compactification functor is a generalization of the Stone-Cech compactification functor $\beta: X \rightarrow \beta X$ that assigns the maximal compactification $\beta X$ to any totally regular space $X$ (i.e., the compactification that corresponds to a maximal compatible proximity). The Stone-Cech functor is one-to-one over the class of first countable spaces [3] but the Smirnov functor is not. Exempli gratia, if $X=(0,1), Y=[0,1)$, and $Z=[0,1]$ are subsets of $\mathbf{R}$ with a proximity induced by a usual metric, then $\sigma X=\sigma Y=\sigma Z=Z=[0,1]$. However, over the class of complete first countable proximity spaces, $\sigma$ is bijective.

Theorem 3 ([14, Theorem 1']). Complete metric spaces with homeomorphic Smirnov compactifications are uniformly equivalent. 
This implies Corollary 4 below. For non-complete spaces we use the following.

Theorem 4 ([14 Corollary 5]). If proximity spaces of countable character have homeomorphic Smirnov compactifications, then their completions are proximate equivalent.

And for metric spaces, this implies uniform equivalence.

Corollary 4. Functor $\lambda$ is one-to-one over a class of complete ultrametric spaces.

The description of properties of functor $\lambda$ goes beyond the scope of the present paper. Here we state only two problems and return to studying subdominant ultrametrics.

Problem 3. Give an entire algebraic description of Boolean algebras $\lambda(X) \in \mathbf{B o o l}_{\Delta}$ that are images of ultrametric spaces under the action of functor $\lambda$.

Problem 4. Describe properties of functor $\lambda$-its relations to categorical operations (such as sums and products, limits of direct and inverse spectra, etc.) and actions of other functors in Umet (the Hausdorff exponential functor, a functor of passing to functional spaces with the metric of uniform convergence, etc.).

Theorem 5. For every metric space $(X, d)$, there are an ultrametric space $\left(u X, d_{u}\right)$ and a non-expanding surjection $u:(X, d) \rightarrow\left(u X, d_{u}\right)$ such that for any nonexpanding map $f:(X, d) \rightarrow(Y, r)$ from $X$ to arbitrary ultrametric spaces $(Y, r)$ there exists a unique nonexpanding map uf: $\left(u X, d_{u}\right) \rightarrow(Y, r)$ that completes the following diagram, i.e., $u f \cdot u=f$ :

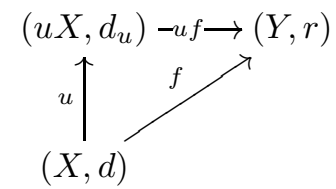

Proof. Let $(X, d)$ be a metric space. Lemma 8 above defines a pseudo-ultrametric $\Delta(x, y)=\inf \{\varepsilon \mid x$ and $y$ are not $\varepsilon$-linkable $\}$. It is easily verified that the binary relation " $x \sim y$ iff $x$ and $y$ are linked", is an equivalence relation. Denote by $[x], u X$, and $u: X \rightarrow u X$ the equivalence class of a point $x$, the quotient space $X / \sim$, and the natural projection $u: X \rightarrow X / \sim$, respectively. Clearly $\Delta(x, y)=0$ iff $x$ and $y$ are linked hence $\Delta(x, y)$ is well-defined on the quotient space and it is an ultrametric there. Denote it by $d_{u}([x],[y])$. Since any pair of points $x$ and $y$ is a $d(x, y)$-chain between them, $d_{u}([x],[y]) \leq d(x, y)$. Therefore the natural projection $u:(X, d) \rightarrow\left(u X, d_{u}\right)$ is non-expanding. The next lemma is obvious.

Lemma 9. Any non-expanding map takes an $\varepsilon$-chain to an $\varepsilon$-chain and, consequently, preserves Cantor connectedness.

Let $(Y, r)$ be an ultrametric space and $f: X \rightarrow Y$ be a non-expanding map. By Lemma 6, for any two points $x$ and $y$ in $X$, the images $f(x)$ and $f(y)$ are not $\varepsilon$-linkable for any $\varepsilon<r(f(x), f(y))$. In view of Lemma[9, $x$ and $y$ are not $\varepsilon$-linkable for any $\varepsilon<r(f(x), f(y))$. Hence $d_{u}([x],[y]) \geq r(f(x), f(y))$. This implies, first, that for $x \sim y, f(x)=f(y)$, i.e., the map $u f: u X \rightarrow Y$, defined as $u f[x]=f(x)$, is well-defined and completes the diagram above. Second, it is non-expanding in view of the inequality $d_{u}([x],[y]) \geq r(f(x), f(y))=r(u f[x], u f[y])$. 
Denote by Metr $\left(\mathbf{M e t r}_{\mathbf{c}}, \mathbf{M e t r}^{\bullet}\right.$ ) a category of all metric spaces (of diameter at most $c$, all metric spaces with a base point) and non-expanding maps (that take a base point to a base point, respectively). Let Ultrametr (Ultrametr $\mathbf{c}_{\mathbf{c}}$, Ultrametr ${ }^{\bullet}$ ) be its subcategory consisting of ultrametric spaces and the same maps. It is known [11], [12], [15], [19] that ultrametric spaces form a full subcategory, which is closed with respect to standard categorical operations (with the only exception - addition of objects in Metr ${ }^{\bullet}$ ). Also, it is closed with respect to actions of basic functors (the completion functor, the Hausdorff exponential functor, a functor of passing to invariant metrics - for spaces acted on by a compact group, functors of passing to various functional spaces with the metric of uniform convergence, etc.) [11], [15]. From the previous theorem one can easily deduce the following.

Theorem 6. The categories Ultrametr, Ultrametr $_{\mathbf{c}}$, and Ultrametr ${ }^{\bullet}$ are epireflective subcategories in $\mathbf{M e t r}, \mathbf{M e t r}_{\mathbf{c}}$ and $\mathbf{M e t r}^{\bullet}$, respectively.

The proof is by a standard categorical discourse.

\section{Non-EXPANDING IMAgeS OF ULtRAMETRIC SPACES}

In [7, 8], F. Hausdorff began a long series of works on metric spaces as images of ultrametric spaces. He proved that any separable metric space $(X, d)$ is an image of a subset $B(X)$ of the Baire space $B_{\aleph_{0}}$ under a continuous open map $f: B(X) \rightarrow X$. K. Morita 20 generalized this to metric spaces of arbitrary weight $\tau$ having replaced $B_{\mathcal{X}_{0}}$ by $B_{\tau}$. A number of topoligists generalized it to more and more wide classes of topological spaces and finally V. Ponomarev 22] proved that the assertion held for all $T_{0}$-spaces of countable character (and only for such spaces!). This hinted that for metric spaces the map $f$ could be improved. Indeed, K. Nagami [21] showed that it could be made compact. Theorem 7 below strengthens this result even more.

Theorem 7. For every cardinal $\tau$, there is a complete ultrametric space $L_{\tau}$ of weight $\tau$ such that any metric space $(X, d)$ of weight $\leq \tau$ is an image of a subspace $L(X) \subset L_{\tau}$ under a non-expanding open map $f: L(X) \rightarrow X$ with compact preimages of points and totally bounded pre-images of compact subsets $K \subset X$.

Proof. By the definition [13], the space $L_{\tau}$ is a set of infinite (in both directions) sequences $\mathbf{a}=\left(\ldots, 0, \alpha_{-m}, \alpha_{-m+1}, \ldots, \alpha_{-1}, \alpha_{0}, \alpha_{1}, \ldots, \alpha_{n}, \ldots\right)$ of ordinals $\alpha_{n}<$ $\omega_{\tau}$ of cardinality less than $\tau$ containing only a finite number of non-zero terms with negative subscript. A metric $d_{L}(\mathbf{a}, \mathbf{b})$ on $L_{\tau}$ is defined as follows: $d_{L}(\mathbf{a}, \mathbf{b})=$ $1 / 2^{n-1}$, where $n=\min \left\{k \mid \alpha_{k} \neq \beta_{k}\right\}$. Clearly, $L_{\tau}$ is a complete ultrametric space of weight $\tau$. A set $L_{\tau}^{0}$ of sequences $\mathbf{a}=\left(\ldots, 0_{-m}, \ldots, 0_{-1}, \alpha_{0}, \alpha_{1}, \ldots, \alpha_{n}, \ldots\right)$ where all terms with negative subscript are equal to zero, can be identified with the generalized Baire space $B_{\tau}$ of weight $\tau$. The metric $d_{L}$ is uniformly equivalent on $L_{\tau}^{0}$ to the standard Baire metric $d_{B}(\mathbf{a}, \mathbf{b})=1 / n$. Actually $L_{\tau}$ generalizes $B_{\tau}$ in the same way as a Laurent series generalizes a Taylor series in complex analysis. If $\operatorname{diam}(X, d) \leq 1$ we can take $L_{\tau}^{0}$ instead of $L_{\tau}$. For this case the theorem is proved in [13, Theorem 1]. For unbounded spaces we refine the proof using the categories Metr $^{\bullet}$ and Ultrametr ${ }^{\bullet}$ (see [13] Theorem 2] and [19]).

Theorem 7 immediately implies the following.

Theorem 8. For every cardinal $\tau$, there is an ultrametric space $L(\tau)$ such that any metric space of weight $\leq \tau$ is an image of $L(\tau)$ under a non-expanding open map. 
To construct $L(\tau)$ consider all pair-wise non-isometric subsets in $L_{\tau}$ containing a point $\mathbf{0}=\left(\ldots, 0_{-m}, \ldots, 0_{-1}, 0_{0}, \ldots, 0_{n}, \ldots\right)$ as a base point. Take a pointed product (= a product in Ultrametr ${ }^{\bullet}[13]$ ) of all these subspaces. In view of [13], [17] it does not exist and natural projections are non-expanding and open [19] Proposition 11].

Problem 5. Construct a universal ultrametric pre-image of smallest weight for all metric spaces of weight $\tau$ under non-expanding open maps.

Note that generally the map $f$ in Theorem 7 cannot be made perfect (pre-images of compact subsets need not be compact) because perfect maps do not augment dimension, whereas all ultrametric spaces are zero-dimensional. At the same time, if a space $(X, d)$ is zero-dimensional, we can refine the covering $\delta_{n}$ to a covering $\zeta_{n}$ consisting of disjoint clopen sets. In this case any point $x$ in $X$ belongs to a unique element of $\zeta_{n}$ for any $n$. This makes the covering map $f: L(X) \rightarrow X$ one-to-one and hence homeomorphic. This strengthens the Morita-de Groot Theorem.

Theorem 9. For any zero-dimensional metric spaces $(X, d)$ of weight $\tau$ there is a subset $L(X) \subset L_{\tau}$ in the universal complete ultrametric space $L_{\tau}$ and a nonexpanding homeomorphism $f: L(X) \rightarrow X$.

Combining Theorem 9 and [10, Lemma VII] we obtain the following.

Corollary 5. Any zero-dimensional metric space $(X, d)$ can be metrized by an ultrametric greater than $d$ without worsening its completeness. In particular, any complete zero-dimensional metric space is metrizable by a complete ultrametric.

A topological space can be metrized by a complete ultrametric if and only if it is metrizable, zero-dimensional and Čech complete.

J. M. Bayod and J. Martínez-Maurica discussed in 1 the problem of existence of a complete ultrametric smaller than $d$ (a complete subdominant ultrametric, in particular) and gave a few examples of spaces that admit and do not admit such an ultrametric. Corollary 6 below partially answers this problem.

Corollary 6. Large proximate zero-dimensional space $(X, d)$ has a complete subdominant ultrametric if and only if it is complete.

If a small proximate zero-dimensional space $(X, d)$ has a complete subdominant ultametric, then $(X, d)$ is complete.

Example 2 below shows that the requirement above is not sufficient for small proximate zero-dimensional spaces.

Example 2. Let $X=\{1,2,5 / 2,3,10 / 3,11 / 3,4, \ldots\}=\{n+m / n \mid m, n \in \mathbf{N}, m<$ $n$ \} be a subset of real line with a usual metric. Then a subdominant ultrametric is $\Delta(x, y)=\max (1 /[x], 1 /[y])$, where $[x]$ denotes an integer part of $x$. Obviously, the space $(X, d)$ is totally unlinked. Moreover, in $\delta(X, d)=0$ although $\operatorname{In} \delta(x, d) \neq 0$ (subsets consisting of even and odd integers are remote but linked). The space $(X, d)$ is complete but $(X, \Delta)$ is not complete, a sequence $x_{n}=n$ is a Cauchy sequence with no limit point in $(X, \Delta)$. However, there is another ultrametric $\Delta^{\prime}$ on $X$ (smaller than $\Delta$ ) such that $\left(X, \Delta^{\prime}\right)$ is complete: $\Delta^{\prime}(x, 1)=1 /[x], \Delta^{\prime}(x, y)=$ $\Delta(x, y)$ for $x \neq 1 \neq y$.

Problem 6. Given a totally unlinked space $(X, d)$, find a necessary and sufficient condition for the existence of a complete ultrametric smaller than $d$ (in particular, for the existence of a complete subdominant ultrametric). 
Problem 7. Given a metric space $(X, d)$, find a necessary and sufficient condition for the completeness of the ultrametrization $\left(u X, d_{u}\right)$.

Definition. For any metric space $(X, d)$ a space $\left(u X, d_{u}\right)$ constructed in Theorem 5 is called an ultrametrization of $(X, d)$.

Unlike the covering map $f: L(X) \rightarrow X$, the ultrametrization map $u:(X, d) \rightarrow$ $\left(u X, d_{u}\right)$ is neither open nor compact (nor closed) in general [19] p. 238]. However, the ultrametrization functor has nice categorical properties. It commutes with sums and products in $\operatorname{Metr}_{\mathbf{c}}, u(x+Y)=u(X)+u(y), u(X \bullet Y)=u(X) \bullet u(Y)$. Moreover, although a sum of spaces in Ultrametr ${ }^{\bullet}$ does not coincide with that in Metr ${ }^{\bullet}$, the property $u(X+Y)=u(X)+u(Y)$ holds in this case as well (also, $u(X \bullet Y)=u(X) \bullet u(Y)$ in Metr and Metr $\left.{ }^{\bullet}\right)$.

Problem 8. Study properties of the ultrametrization functor, in particular, its relation to other functors, its action on particular classes of spaces and maps, etc.

For the last two decades, the theory of ultrametric spaces has found strong relations to geometry of Euclidean and Hilbert space, Lebesgue measure and integral theory, the theory of Boolean algebras and lattice theory, set theory and foundations, category theory and topoi, $p$-adic analysis and $p$-adic functional analysis, physics, biology, and computer science (see 15-19] and references therein). Compare Theorem 2 above with the following.

Theorem ([15]). A category Ultrametr is isomorphic to a category $\mathbf{L A T}^{*}$ of complete, atomic, tree-like, real-graduated lattices and isotonic, semi-continuous, non-extensive maps.

This means, in particular, that for any ultrametric space $(X, d)$ there exists a unique lattice $\boldsymbol{L}(X)$ in $\mathbf{L} \mathbf{A} \mathbf{T}^{*}$, and for any lattice $L$ in $\mathbf{L} \mathbf{A} \mathbf{T}^{*}$ there exists a unique ultrametric space $\boldsymbol{A}(L)$ such that $\boldsymbol{A}(\boldsymbol{L}(X))=X, \boldsymbol{L}(\boldsymbol{A}(L))=L$, where the first equality is an isometry of metric spaces whereas the second one is an isomorphism of lattices (see [15] for proofs and details). The paper [15] describes in detail the properties of the isomorphism functor $\boldsymbol{L}:$ Ultrametr $\rightarrow \mathbf{L A T}^{*}$ - its relations to categorical operation and actions of other functors, and its action on the particular classes of spaces (bounded, totally bounded, complete, spherically complete, compact, etc.). Composing functors $u$ and $\boldsymbol{L}$ we get a functor $U \bullet L:$ Metr $\rightarrow \mathbf{L A T}^{*}$, which provides us with a lattice theoretical classification of general metric spaces. This will be described in a separate paper.

\section{ADDED IN PROOF}

The Hausdorff-Bayod Problem was discussed in the interesting survey [29] among other open problems. Some of them are also solved here.

\section{REFERENCES}

1. J. M. Bayod, J. Martínez-Maurica, Subdominant ultrametrics, Proc. AMS, 109:3 (1990), 829834. MR 90k:54038

2. J. M. Bayod, The space $l_{1}(K)$ is not ultrametrizable, in "p-adic Functional Analysis", pp. 221-225 (Lecture Notes in Pure \& Applied Math. 137), Marcel Dekker, NY, 1992. MR 93a: 46140

3. E. Čech, On bicompact spaces, Ann. of Math. 38 (1937), 823-844.

4. V. A. Efremovich, Proximity geometry, Math. Sbornik 31 (1952), 189-200.

5. R. Engelking, General topology, Warszawa, PWN, 1977. MR 58:18316b 
6. J. de Groot, Non-Archimedean metrics in topology, Proc. AMS 7:6 (1956) 948-956.

7. F. Hausdorff, Erweiterung einer Homeomorphie, Fund. Math. 16 (1930), 353-360.

8. _ U Über innere Abbildungen, Fund. Math. 23 (1934), 279-291.

9. _ Set Theory, Leipzig, 1914 (Russian edition revised and completed by Paul Alexandroff and Andrei Kolmogoroff, Moscow, 1937).

10. A. J. Lemin, Proximity on isosceles spaces, Russian Math. Surveys 39:1 (1984), 143-144.

11. - On stability of the property of a space being isosceles, Russian Math. Surveys 39:5 (1984), 283-284.

12. _ Transition functor to a function space in the uniform topology, Russian Math. Surveys 40:6 (1985), 133-134.

13. - Inverse images of metric spaces under non-expanding open mappings, Russian Math. Surveys 43:3 (1988), 214-215.

14. The Smirnov compactification functor is one-to-one over the class of complete first countable spaces, Topology and its applications 38 (1991) 201-204. MR 92b:54057

15. The category of ultrametric spaces is isomorphic to the category of complete, atomic, tree-like, and real graduated lattices LAT*, Algebra Universalis (to appear).

16. _ Isometric embedding of ultrametric (non-Archimedean) spaces in Hilbert space and Lebesgue space, in "p-adic Functional Analysis" (Lecture Notes in Pure \& Applied Math. 222), Marcel Dekker, 2001, pp. 203-218. MR 2002d:46058

17. U Ultrametric spaces and Boolean algebras, "5 $5^{\text {th }}$ international conference "Topology and its Applications", Dubrovnik June 1990, Abstracts", Zagreb 1990, p. 49.

18. A. J. Lemin and V. Lemin, On a universal ultrametric space, Topology and its Applications 103 (2000), 339-345. MR 2001b:54035

19. V. Lemin, Finite ultrametric spaces and computer science, in: "Categorical Perspectives", ed. Jürgen Koslowski, Austin Melton (Trends in Mathematics, v. 16), Birkhäuser-Verlag, Boston, Basel, Berlin, 2001, pp. 219-242.

20. K. Morita, Normal families and dimension theory for metric spaces, Math. Ann. 128 (1954), $350-362$.

21. K. Nagami, A note on Hausdorff spaces with star-finite property. I, II, Proc. Japan Acad. 37 (1961), 131-134, 189-192. MR 26:1852

22. V. I. Ponomarev, Axioms of countability and continuous maps, Bull. Acad. Pol. Sci. 8:3 (1960), 127-133. MR 22:7109

23. R. Rammal, G. Toulouse, and M. A. Virasoro, Ultrametricity for physicists, Rev. Modern Physics 58 (1986), 765-788. MR 87k:82105

24. Yu. M. Smirnov, On proximity spaces, Math. Sbornik 31 (1952), 543-574 (in Russian), AMS Trans. Ser. 2, 38, 5-35.

25. - On completeness of proximity spaces, Proceedings of Moscow Math. Soc. 3 (1954), 271-306.

26. $ـ$ On the dimension of proximity spaces, Math. Sbornik 38 (1956), 283-302 (in Russian), AMS. Trans. Ser. 2, 38, 37-73. MR 18:497b

27. M. Stone, Applications of the theory of Boolean rings to general topology, Trans. AMS 41 (1937), 375-481.

28. J. E. Vaughan, Universal ultrametric spaces of smallest weight, Topology Proceedings 24 (2001), 611-619.

29. S. Watson, The classification of metrics and multivariate statistical analysis, Topology and its Applications 99 (1999), 237-261. MR 2000i:54038

Department of Mathematics, Moscow State University of Civil Engineering, 26 YaroSLavskoe Highway, Moscow 129337, Russia

E-mail address: alex_lemin@hotmail.com 